\title{
Letter
}

\section{Triple-parton scatterings in proton-nucleus collisions at high energies}

\author{
David d'Enterria ${ }^{1, \mathrm{a}}$, Alexander M. Snigirev ${ }^{2,3, \mathrm{~b}}$ \\ ${ }^{1}$ EP Department, CERN, 1211 Geneva, Switzerland \\ 2 Skobeltsyn Institute of Nuclear Physics, Lomonosov Moscow State University, 119991 Moscow, Russia \\ ${ }^{3}$ Bogoliubov Laboratory of Theoretical Physics, JINR, 141980 Dubna, Russia
}

Received: 30 June 2017 / Accepted: 4 March 2018 / Published online: 8 May 2018

(C) The Author(s) 2018

\begin{abstract}
A generic expression to compute triple-parton scattering (TPS) cross sections in high-energy protonnucleus (pA) collisions is derived as a function of the corresponding single-parton cross sections and an effective parameter encoding the transverse parton profile of the proton. The TPS cross sections are enhanced by a factor of about $9 \mathrm{~A} \simeq 2000 \mathrm{in} \mathrm{pPb}$ as compared to those in proton-nucleon collisions at the same center-of-mass energy. Estimates for triple charm $(\mathrm{c} \overline{\mathrm{c}})$ and bottom $(\mathrm{b} \overline{\mathrm{b}})$ production in $\mathrm{pPb}$ collisions at LHC and FCC energies are presented based on nextto-next-to-leading-order calculations for $\mathrm{c} \overline{\mathrm{c}}$ and $\mathrm{b} \overline{\mathrm{b}}$ singleparton cross sections. At $\sqrt{s_{\mathrm{NN}}}=8.8 \mathrm{TeV}$, about $10 \%$ of the $\mathrm{pPb}$ events have three $\mathrm{c} \overline{\mathrm{c}}$ pairs produced in separate partonic interactions. At $\sqrt{s_{\mathrm{NN}}}=63 \mathrm{TeV}$, the $\mathrm{pPb}$ cross sections for triple- $J / \psi$ and triple-b $\bar{b}$ are $\mathscr{O}(1-10 \mathrm{mb})$. In the most energetic collisions of cosmic rays in the upper atmosphere, equivalent to $\sqrt{s_{\mathrm{NN}}} \approx 400 \mathrm{TeV}$, the TPS ce cross section equals the total $\mathrm{p}-\mathrm{Air}$ inelastic cross section.
\end{abstract}

\section{Introduction}

The extended nature of hadronic systems and their growing parton density when probed at increasingly higher collision energies, makes it possible to produce multiple particles with large transverse momentum and/or mass $\left(\sqrt{\mathrm{p}_{\mathrm{T}}^{2}+\mathrm{m}^{2}} \gtrsim 3 \mathrm{GeV}\right)$ in independent multiparton interactions (MPIs) in high-energy proton-(anti)proton (pp, p $\bar{p}$ ) collisions [1-5]. Many experimental final states - involving the concurrent production of heavy-quarks, quarkonia, jets, and gauge bosons - have been found to be consistent with double parton scatterings (DPS) processes at Tevatron (see e.g. [6]) and the LHC (see e.g. [7-9] for a selection of the latest

\footnotetext{
a e-mail: david.d'enterria@cern.ch

be-mail: snigirev@lav01.sinp.msu.ru
}

results). Multiple hard parton interaction rates depend chiefly on the transverse overlap of the matter densities of the colliding hadrons, and provide valuable information on (1) the badly known 3D parton profile of the proton, (2) the unknown energy evolution of the parton density as a function of impact parameter (b), and (3) the role of many-parton correlations in the hadronic wave functions [10]. In our previous work [1114], we highlighted the importance of studying DPS also in proton-nucleus (pA) and nucleus-nucleus (AA) collisions, as a complementary means to improve our understanding of hard MPIs in pp collisions. The larger transverse parton density in a nucleus (with A nucleons) compared to that of a proton, results in enhanced DPS cross sections coming from interactions where the two partons of the nucleus belong to the same or to two different nucleons, providing thereby useful information on the underlying multiparton dynamics [1121].

The possibility of triple-parton scatterings (TPS) in hadronic collisions has also been considered in the literature [22-26], and estimates of their expected cross sections have been recently provided for pp collisions [27]. In this paper, we extend our latest work and derive for the first time quantitative estimates of the cross sections for observing three separate hard interactions in a pA collision through a factorized formula which depends on the underlying single-parton scattering (SPS) cross sections normalized by the square of an effective cross section $\sigma_{\text {eff, TPS }}$, characterizing the transverse area of triple-partonic interactions, that is closely related to the DPS-equivalent $\sigma_{\text {eff,DPS }}$ parameter [27]. The paper is organized as follows. In Sect. 2, we review the theoretical expression for TPS cross sections in generic hadron-hadron collisions, first expressed as a convolution of SPS cross sections and generalized parton densities dependent on parton fractional momentum $x$, virtuality $Q^{2}$, and impact parameter $b$, and then in their factorized form as a function of $\sigma_{\text {eff,TPS }}$. In Sect. 3, a generic expression for TPS cross sections in pA 
collisions is presented based on realistic parametrizations of the nuclear transverse profile. As concrete numerical examples, Sect. 4 provides estimates for triple charm (c $\overline{\mathbf{c}})$ and bottom $(b \bar{b})$ cross sections from independent parton scatterings in proton-lead $(\mathrm{pPb})$ collisions at the LHC and future circular collider (FCC) [28] energies, as well as in protonair collisions at the highest energies observed so far, based on next-to-next-to-leading-order (NNLO) calculations of the corresponding SPS cross sections. The main conclusions are summarized in Sect. 5.

\section{Triple-parton-scattering cross sections in hadron-hadron collisions}

In a generic hadronic collision, the inclusive TPS cross section from three independent hard parton scatterings $\left(h h^{\prime} \rightarrow\right.$ $a b c$ ) can be written as a convolution of generalized parton distribution functions (PDF) and elementary cross sections summed over all involved partons [22-27,29]

$$
\begin{aligned}
\sigma_{h h^{\prime} \rightarrow a b c}^{\mathrm{TPS}} & \frac{m}{3 !} \sum_{i, j, k, l, m, n} \int \Gamma_{h}^{i j k}\left(x_{1}, x_{2}, x_{3} ; \mathbf{b}_{\mathbf{1}}, \mathbf{b}_{\mathbf{2}}, \mathbf{b}_{\mathbf{3}} ; Q_{1}^{2}, Q_{2}^{2}, Q_{3}^{2}\right) \\
& \times \hat{\sigma}_{a}^{i l}\left(x_{1}, x_{1}^{\prime}, Q_{1}^{2}\right) \hat{\sigma}_{b}^{j m}\left(x_{2}, x_{2}^{\prime}, Q_{2}^{2}\right) \hat{\sigma}_{c}^{k n}\left(x_{3}, x_{3}^{\prime}, Q_{3}^{2}\right) \\
& \times \Gamma_{h^{\prime}}^{l m n}\left(x_{1}^{\prime}, x_{2}^{\prime}, x_{3}^{\prime} ; \mathbf{b}_{1}-\mathbf{b}, \mathbf{b}_{2}-\mathbf{b}, \mathbf{b}_{3}-\mathbf{b} ; Q_{1}^{2}, Q_{2}^{2}, Q_{3}^{2}\right) \\
& \times d x_{1} d x_{2} d x_{3} d x_{1}^{\prime} d x_{2}^{\prime} d x_{3}^{\prime} d^{2} b_{1} d^{2} b_{2} d^{2} b_{3} d^{2} b .
\end{aligned}
$$

Here, $\Gamma_{h}^{i j k}\left(x_{1}, x_{2}, x_{3} ; \mathbf{b}_{1}, \mathbf{b}_{2}, \mathbf{b}_{3} ; Q_{1}^{2}, Q_{2}^{2}, Q_{3}^{2}\right)$ are the tripleparton distribution functions, depending on the momentum fractions $x_{1}, x_{2}, x_{3}$ at transverse positions $\mathbf{b}_{\mathbf{1}}, \mathbf{b}_{\mathbf{2}}, \mathbf{b}_{\mathbf{3}}$ of the three partons $i, j, k$, producing final states $a, b, c$ at energy scales $Q_{1}, Q_{2}, Q_{3}$, with subprocess cross sections $\hat{\sigma}_{a}^{i l}, \hat{\sigma}_{b}^{j m}$, $\hat{\sigma}_{c}^{k n}$. The combinatorial prefactor $m / 3$ ! takes into account the different cases of (indistinguishable or not) final states: $m=1$ if $a=b=c ; m=3$ if $a=b$, or $a=c$, or $b=c$; and $m=6$ if a, b, c are different. The triple-parton distribution functions $\Gamma_{h}^{i j k}\left(x_{1}, x_{2}, x_{3} ; \mathbf{b}_{1}, \mathbf{b}_{2}, \mathbf{b}_{3} ; Q_{1}^{2}, Q_{2}^{2}, Q_{3}^{2}\right)$ encode all the parton structure information of relevance for TPS.

Without any loss of generality, any TPS cross section can always be expressed in a very economical form as a triple product of single inclusive cross sections,

$\sigma_{h h^{\prime} \rightarrow a}^{\mathrm{SPS}}=\sum_{i, k} \int D_{h}^{i}\left(x_{1} ; Q_{1}^{2}\right) \hat{\sigma}_{a}^{i k}\left(x_{1}, x_{1}^{\prime}\right) D_{h^{\prime}}^{k}\left(x_{1}^{\prime} ; Q_{1}^{2}\right) \mathrm{d} x_{1} \mathrm{~d} x_{1}^{\prime}$,

normalized by the square of an effective TPS cross section, namely

$\sigma_{h h^{\prime} \rightarrow a b c}^{\mathrm{TPS}}=\left(\frac{m}{3 !}\right) \frac{\sigma_{h h^{\prime} \rightarrow a}^{\mathrm{SPS}} \cdot \sigma_{h h^{\prime} \rightarrow b}^{\mathrm{SPS}} \cdot \sigma_{h h^{\prime} \rightarrow c}^{\mathrm{SPS}}}{\sigma_{\mathrm{eff}, \mathrm{TPS}}^{2}}$, where $\sigma_{\text {eff,TPS }}$ encodes all the unknowns related to the generalized PDFs and their correlations in space, color, flavor, $\mathrm{p}_{\mathrm{T}}, \ldots$ The value of $\sigma_{\text {eff, TPS }}$ can be estimated theoretically making a few common approximations. First, the triple-PDF are commonly assumed to be factorizable in terms of longitudinal and transverse components, i.e.

$$
\begin{aligned}
& \Gamma_{h}^{i j k}\left(x_{1}, x_{2}, x_{3} ; \mathbf{b}_{1}, \mathbf{b}_{2}, \mathbf{b}_{3} ; Q_{1}^{2}, Q_{2}^{2}, Q_{3}^{2}\right) \\
& \quad=D_{h}^{i j k}\left(x_{1}, x_{2}, x_{3} ; Q_{1}^{2}, Q_{2}^{2}, Q_{3}^{2}\right) f\left(\mathbf{b}_{1}\right) f\left(\mathbf{b}_{2}\right) f\left(\mathbf{b}_{3}\right),
\end{aligned}
$$

where $f\left(\mathbf{b}_{1}\right)$ describes the transverse parton density of the hadron, often considered a universal function for all types of partons, from which the corresponding hadron-hadron overlap function is derived:

$T(\mathbf{b})=\int f\left(\mathbf{b}_{1}\right) f\left(\mathbf{b}_{1}-\mathbf{b}\right) d^{2} b_{1}$.

Making the further assumption that the longitudinal components reduce to the product of independent single PDF, $D_{h}^{i j k}\left(x_{1}, x_{2}, x_{3} ; Q_{1}^{2}, Q_{2}^{2}, Q_{3}^{2}\right)=D_{h}^{i}\left(x_{1} ; Q_{1}^{2}\right) D_{h}^{j}\left(x_{2} ; Q_{2}^{2}\right)$ $D_{h}^{k}\left(x_{3} ; Q_{3}^{2}\right)$, the effective TPS cross section has a very simple geometric interpretation as the inverse of the cube of the integral of the hadron-hadron overlap function:

$\sigma_{\mathrm{eff}, \mathrm{TPS}}^{2}=\left\{\int d^{2} b T^{3}(\mathbf{b})\right\}^{-1}$,

which is closely related to the similar quantity,

$\sigma_{\mathrm{eff}, \mathrm{DPS}}=\left\{\int d^{2} b T^{2}(\mathbf{b})\right\}^{-1}$,

determined in DPS measurements. In the proton-proton case, making use of Eqs. (5), (6) and (7), for a wide range of proton transverse parton profiles $f(\mathbf{b})$, we found a simple relationship between the effective DPS and TPS cross sections:

$\sigma_{\mathrm{eff}, \mathrm{TPS}}=(0.82 \pm 0.11) \cdot \sigma_{\mathrm{eff}, \mathrm{DPS}}$

which, for the typical $\sigma_{\text {eff,DPS }}=15 \pm 5 \mathrm{mb}$ values extracted from a broad range of DPS measurements at Tevatron [6] and LHC [4, 6-9], translates into

$\sigma_{\mathrm{eff}, \mathrm{TPS}}=12.5 \pm 4.5 \mathrm{mb}$.

This data-driven numerical value allows for the computation of any TPS cross section in pp collisions via Eq. (3), once the corresponding SPS cross section, Eq. (2), is known.

In the next section, we extend and exploit these results for the $\mathrm{pA}$ case. We note that, since we start off with a numerical value for $\sigma_{\text {eff,DPs }}$ that is directly obtained from data, our approach effectively takes into account effects which go beyond pure geometrical quantities computed in terms of transverse overlap of parton densities. Indeed, the fact that the experimentally extracted $\sigma_{\text {eff,DPS }}=15 \pm 5 \mathrm{mb}$ values are about a factor of 2 smaller (i.e., that the DPS cross 
sections are about twice larger) than expected from Eq. (7) for a "naive" proton profile, is indicative of the presence of "beyond mean-field" effects, including e.g. parton correlations in momentum, flavor, color, $x, \ldots$ Perturbative partonic correlations due to 1-to-2 parton splittings have been discussed for the DPS case in [30,31] in pp collisions and in [21] for pA collisions. The first attempt to estimate those for TPS in pp collisions was done in [26]. As we will see later, however, any such potential effects are of less relevance in pA than in pp collisions, since the majority of the TPS yields for the former arise from interactions among different "target" nucleons for which any potential partonic correlations are of smaller magnitude than in the pp case.

\section{Triple-parton-scattering cross sections in proton-nucleus collisions}

The first theoretical analysis of double- and triple-parton scattering cross sections in pA collisions was done in $[15,16]$, where large enhancements of the DPS and TPS yields were anticipated. Here we focus on the derivation of a compact "pocket formula" for TPS scattering in pA collisions, particularly useful for phenomenological and numerical applications. The starting point of our approach is to remember that the parton flux in $\mathrm{pA}$ compared to $\mathrm{pp}$ is enhanced by the nucleon number $\mathrm{A}$ and, modulo shadowing effects in the nuclear PDF [32], the single-parton cross section for any hard process is that of proton-nucleon $(\mathrm{pN})$ collisions (with $\mathrm{N}=\mathrm{p}, \mathrm{n}$ including their appropriate relative fraction in the nucleus) scaled by the factor A [33],

$\sigma_{\mathrm{pA} \rightarrow \mathrm{abc}}^{\mathrm{SPS}}=\sigma_{\mathrm{pN} \rightarrow \mathrm{abc}}^{\mathrm{SPS}} \int \mathrm{d}^{2} \mathrm{bT}_{\mathrm{A}}(\mathbf{b})=\mathrm{A} \cdot \sigma_{\mathrm{pN} \rightarrow \mathrm{abc}}^{\mathrm{SPS}} \cdot$

Here $\mathrm{T}_{\mathrm{A}}(\mathbf{b})=\int \mathrm{f}_{\mathrm{A}}\left(\sqrt{\mathrm{r}^{2}+\mathrm{z}^{2}}\right) d \mathrm{z}$ is the nuclear thickness function given by the integral of the nuclear parton density function (commonly parametrized in terms of a "WoodsSaxon" Fermi-Dirac distribution [34]) over the longitudinal direction with respect to the impact parameter $\mathbf{b}$ between the colliding proton and nucleus, normalized to $\int \mathrm{d}^{2} \mathrm{~b} \mathrm{~T}_{\mathrm{A}}(\mathbf{b})=$ A. In order to obtain a TPS "pocket formula" of the form of Eq. (3) for pA collisions, we follow the approach developed in our previous work for the DPS case [11-14]. The TPS $\mathrm{pA}$ cross section is thus obtained from the sum of three contributions:

- A "pure TPS" cross section, given by Eq. (3) for pN collisions scaled by A, namely:

$\sigma_{\mathrm{pA} \rightarrow \mathrm{abc}}^{\mathrm{TPS} 1}=\mathrm{A} \cdot \sigma_{\mathrm{pN} \rightarrow \mathrm{abc}}^{\mathrm{TPS}} \cdot$

- A second contribution, involving interactions of partons from two different nucleons in the nucleus, depending on the square of $\mathrm{T}_{\mathrm{A}}$,

$$
\begin{aligned}
\sigma_{\mathrm{pA} \rightarrow \mathrm{abc}}^{\mathrm{TPS}, 2} & =\sigma_{\mathrm{pN} \rightarrow \mathrm{abc}}^{\mathrm{TPS}} \cdot 3 \frac{\sigma_{\mathrm{eff}, \mathrm{TPS}}^{2}}{\sigma_{\mathrm{eff}, \mathrm{DPS}}} \mathrm{F}_{\mathrm{pA}}, \text { with } \\
\mathrm{F}_{\mathrm{pA}} & =\frac{\mathrm{A}-1}{\mathrm{~A}} \int \mathrm{d}^{2} \mathrm{bT}_{\mathrm{A}}^{2}(\mathbf{b}),
\end{aligned}
$$

where the factor $(\mathrm{A}-1) / \mathrm{A}$ is introduced to account for the difference between the number of nucleon pairs and the number of different nucleon pairs.

- A third term, involving interactions among partons from three different nucleons, depending on the cube of $\mathrm{T}_{\mathrm{A}}$,

$$
\begin{aligned}
\sigma_{\mathrm{pA} \rightarrow \mathrm{abc}}^{\mathrm{TPS},} & =\sigma_{\mathrm{pN} \rightarrow \mathrm{abc}}^{\mathrm{TPS}} \cdot \sigma_{\mathrm{eff}, \mathrm{TPS}}^{2} \cdot \mathrm{C}_{\mathrm{pA}}, \text { with } \\
\mathrm{C}_{\mathrm{pA}} & =\frac{(\mathrm{A}-1)(\mathrm{A}-2)}{\mathrm{A}^{2}} \int \mathrm{d}^{2} \mathrm{~b} \mathrm{~T}_{\mathrm{A}}^{3}(\mathrm{~b}) .
\end{aligned}
$$

The factor $(\mathrm{A}-1)(\mathrm{A}-2) / \mathrm{A}^{2}$ is introduced to take into account the difference between the total number of nucleon TPS and that of different nucleon TPS.

The inclusive TPS cross section for three hard parton subprocesses $a, b$, and $c$ in $\mathrm{pA}$ collisions is thus obtained from the sum of the three terms (11), (12), and (14):

$\sigma_{\mathrm{pA} \rightarrow \mathrm{abc}}^{\mathrm{TPS}}=\mathrm{A} \sigma_{\mathrm{pN} \rightarrow \mathrm{abc}}^{\mathrm{TPS}}\left[1+3 \frac{\sigma_{\mathrm{eff}, \mathrm{TPS}}^{2}}{\sigma_{\mathrm{eff}, \mathrm{DPS}}} \frac{\mathrm{F}_{\mathrm{pA}}}{\mathrm{A}}+\sigma_{\mathrm{eff}, \mathrm{TPS}}^{2} \frac{\mathrm{C}_{\mathrm{pA}}}{\mathrm{A}}\right]$,

which is enhanced by the factor in parentheses compared to the corresponding TPS cross section in $\mathrm{pN}$ collisions scaled by A. The analytical (unintegrated) expression for TPS cross sections in proton-nucleus collisions was first derived in [15], but our compact expression (16) provides a more useful formula to easily derive numerical TPS estimates for any pA system for phenomenological purposes. The value of this enhancement factor, as well as the relative role of each one of the three TPS components, can be obtained for $\mathrm{pPb}$ evaluating the integrals (13) and (15) using the standard Fermi-Dirac spatial density for the lead nucleus $(\mathrm{A}=208$, radius $\mathrm{R}_{\mathrm{A}}=6.36 \mathrm{fm}$, and surface thickness $a=0.54 \mathrm{fm}$ ) [34]. The first integral is identical to the overlap function at zero impact parameter for the corresponding AA collision, $\mathrm{F}_{\mathrm{pA}}=(\mathrm{A}-1) / \mathrm{A} \mathrm{T}_{\mathrm{AA}}(0)=30.25 \mathrm{mb}^{-1}[11-14]$. The second one can be obtained by means of a Glauber Monte Carlo (MC) [33] and amounts to $\mathrm{C}_{\mathrm{pA}}=4.75 \mathrm{mb}^{-2}$. From the relationship (8) between the effective DPS and TPS cross sections, and the experimental $\sigma_{\text {eff,DPs }}=15 \pm 5 \mathrm{mb}$ value [4, 6-9], we can finally determine the relative importance for $\mathrm{pPb}$ of the three TPS terms of Eq. (16): $\sigma_{\mathrm{pA} \rightarrow \mathrm{abc}}^{\mathrm{TPS}, 1}: \sigma_{\mathrm{pA} \rightarrow \mathrm{abc}}^{\mathrm{TPS}, 2}:$ $\sigma_{\mathrm{pA} \rightarrow \mathrm{abc}}^{\mathrm{TPS}, 3}=1: 4.54: 3.56$. Namely, in pPb collisions, $10 \%$ of the TPS yields come from partonic interactions within just one nucleon of the lead nucleus, 50\% involve scatterings within two nucleons, and $40 \%$ come from partonic interac- 
tions in three different $\mathrm{Pb}$ nucleons. The fact that, for heavy nuclei, the second and third terms of Eq. (16) are much larger than the first one was first pointed out in Ref. [15], and we provide here an exact numerical evaluation for the $\mathrm{pPb}$ case. The sum of the three contributions in Eq. (16) amounts to 9.1, namely the TPS cross sections in $\mathrm{pPb}$ are about nine times larger than the naive expectation based on A-scaling of the corresponding pN TPS cross sections, Eq. (11). We note that in the DPS case the equivalent $\mathrm{pA}$ enhancement factor was $\left[1+\sigma_{\text {eff,DPS }} \mathrm{F}_{\mathrm{pA}} / \mathrm{A}\right] \simeq 3[11-14]$. The final formula for TPS in the proton-nucleus case reads

$\sigma_{\mathrm{pA} \rightarrow \mathrm{abc}}^{\mathrm{TP}}=\left(\frac{m}{6}\right) \frac{\sigma_{\mathrm{pN} \rightarrow \mathrm{a}}^{\mathrm{SPS}} \cdot \sigma_{\mathrm{pN} \rightarrow \mathrm{b}}^{\mathrm{SPS}} \cdot \sigma_{\mathrm{pN} \rightarrow \mathrm{c}}^{\mathrm{SPS}}}{\sigma_{\mathrm{eff}, \mathrm{TPS}, \mathrm{pA}}^{2}}$,

where the effective TPS pA cross section in the denominator depends on the effective pp one and on pure geometric quantities directly derivable from the well-known nuclear transverse profile:

$$
\begin{aligned}
\sigma_{\text {eff }, \mathrm{TPS}, \mathrm{pA}}^{2} & =\left\{\mathrm{A} / \sigma_{\mathrm{eff}, \mathrm{TPS}}^{2}+2.46 \mathrm{~F}_{\mathrm{pA}} / \sigma_{\mathrm{eff}, \mathrm{TPS}}+\mathrm{C}_{\mathrm{pA}}\right\}^{-1} \\
& =\left\{\mathrm{A} / 156 .+\mathrm{F}_{\mathrm{pA}}\left[\mathrm{mb}^{-1}\right] / 5 .+\mathrm{C}_{\mathrm{pA}}\left[\mathrm{mb}^{-2}\right]\right\}^{-1},
\end{aligned}
$$

where the latter equality is obtained using Eqs. (8)-(9). The effective TPS cross section in the $\mathrm{pPb}$ case amounts thereby to $\sigma_{\text {eff, } \mathrm{TPS}, \mathrm{pA}}=0.29 \pm 0.05 \mathrm{mb}$. This value is very robust with respect to the parametrization of the underlying proton and nucleus transverse profiles. Indeed, by using simplified Gaussian proton and nucleus transverse densities, all relevant factors in Eq. (16) can be analytically calculated, and the effective TPS pA cross section can be simply written as a function of the proton and nucleus radii: $\sigma_{\text {eff, TPS,pA }}^{2}=$ $3 / 4 \sigma_{\text {eff,DPS }}^{2} /\left\{\mathrm{A}\left[1+9 / 2 \mathrm{~A}\left(\mathrm{r}_{\mathrm{p}} / \mathrm{R}_{\mathrm{A}}\right)^{2}+4 \mathrm{~A}^{2}\left(\mathrm{r}_{\mathrm{p}} / \mathrm{R}_{\mathrm{A}}\right)^{4}\right]\right\}$, which amounts to $\sigma_{\text {eff, TPS,pA }} \simeq 0.28 \mathrm{mb}$ (fixing $r_{p}$ so as to $\sigma_{\text {eff,DPS }}=$ $15 \mathrm{mb}$ ), in perfect agreement with our more accurate estimate above.

\section{Triple c $\bar{c}$ and $b \bar{b}$ production cross sections in $\mathbf{p A}$ collisions}

As concrete numerical examples of our calculations, following our previous similar pp study [27], we compute the charm $(\mathrm{pPb} \rightarrow \mathrm{c} \overline{\mathrm{c}}+\mathrm{X})$ and bottom $(\mathrm{pPb} \rightarrow \mathrm{b} \overline{\mathrm{b}}+\mathrm{X})$ TPS cross sections first at the LHC and FCC center-of-mass (c.m.) energies, and then also in proton-air collisions of relevance for ultra-high-energy cosmic-ray collisions in the atmosphere. These processes are dominated by gluon-gluon scattering $g g \rightarrow \mathrm{QQ}$ at low parton fractional momentum $x$, for which the DPS and TPS mechanisms have a growing contribution to the total inclusive production at increasing c.m. energy. This expectation has been discussed for the DPS case in [35], and we extend those studies to the TPS case here. The TPS heavyquark cross sections are computed via Eq. (17) for $m=1$, i.e. $\sigma_{\mathrm{pPb} \rightarrow \mathrm{c} \bar{c}, \mathrm{~b} \overline{\mathrm{b}}}^{\mathrm{TPS}}=\left(\sigma_{\mathrm{pN} \rightarrow \mathrm{c} \overline{\mathrm{c}}, \mathrm{b} \overline{\mathrm{b}}}^{\mathrm{SPS}}\right)^{3} /\left(6 \sigma_{\mathrm{eff}, \mathrm{TPS}, \mathrm{pA}}^{2}\right)$ with $\sigma_{\text {eff, } \mathrm{TPS}, \mathrm{pA}}$ given by (18), and $\sigma_{\mathrm{pN} \rightarrow \mathrm{c} \bar{c}, \mathrm{~b} \overline{\mathrm{b}}}^{\mathrm{SPS}}$ is calculated via Eq. (2) at NNLO accuracy using a modified version [36] of the Top++ (v2.0) code [37]. Top++ is run with $\mathrm{N}_{\mathrm{f}}=3,4$ light flavors, charm and bottom pole masses set to $\mathrm{m}_{\mathrm{c}, \mathrm{b}}=1.67,4.66 \mathrm{GeV}$ [38], default renormalization and factorization scales set to $\mu_{\mathrm{R}}=\mu_{\mathrm{F}}=2 \mathrm{~m}_{\mathrm{c}, \mathrm{b}}$, and using the NNLO ABMP6 PDF of the proton [39] and the nuclear PDF modification factors of the $\mathrm{Pb}$ nucleus given by EPS09-NLO [32]. The PDF uncertainties include those from the proton and nucleus, as obtained from the corresponding 28 (30) eigenvalues of the ABMP16 (EPS09) sets, combined in quadrature. The dominant uncertainty is that linked to the theoretical scale choice, which is estimated by modifying $\mu_{\mathrm{R}}$ and $\mu_{\mathrm{F}}$ within a factor of 2 . In the pp case, such a theoretical NNLO setup yields SPS heavyquark cross sections which are larger by up to $20 \%$ compared to the NLO $[40,41]$ predictions at the LHC, reaching a better agreement with the experimental data, and showing a much reduced scale uncertainty $( \pm 50 \%, \pm 15 \%$ for $c \bar{c}, b \bar{b})$ [36]. In the $\mathrm{pPb}$ case, the inclusion of EPS09 nuclear shadowing reduces moderately the total charm and bottom cross sections in pN compared to pp collisions, by about $10 \%$ (13\%) and $5 \%(10 \%)$ at the LHC (FCC). Since the TPS pPb cross section go as the cube of $\sigma_{\mathrm{pN} \rightarrow \mathrm{QQ}}^{\mathrm{SPS}}$, the impact of shadowing is amplified and leads to $15-35 \%$ reductions with respect to the result obtained if one used the pp (instead of the $\mathrm{pN}$ ) SPS cross section in Eq. (17). At $\sqrt{s}=5.02 \mathrm{TeV}$, our theoretical SPS prediction $\left(\sigma_{\mathrm{pPb} \rightarrow \mathrm{c} \overline{\mathrm{C}}}^{\mathrm{SPS}, \mathrm{NLO}}=650 \pm 290_{\mathrm{sc}} \pm 60_{\mathrm{pdf}} \mathrm{mb}\right)$ agrees well with the ALICE total D-meson measurement [42] extrapolated using [40] to a total charm cross section $\left(\sigma_{\mathrm{pPb} \rightarrow \mathrm{c} \overline{\mathrm{c}}}^{\mathrm{ALICE}}=640 \pm 60_{\text {stat }}+\left.\left.60\right|_{-110}\right|_{\text {syst }} \mathrm{mb}\right.$, Fig. 1 left $)$.

Table 1 collects the heavy-quark cross sections and associated uncertainties predicted in $\mathrm{pPb}$ collisions at the nominal LHC and FCC c.m. energies. The large SPS cē cross

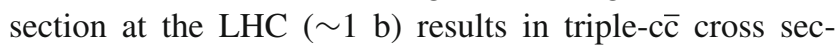
tions from independent parton scatterings amounting to about $20 \%$ of the inclusive charm yields. Since the total inelastic $\mathrm{pPb}$ cross sections are $\sigma_{\mathrm{pPb}}=2.2,2.4 \mathrm{~b}$ at $\sqrt{s_{\mathrm{NN}}}=8.8$ and $63 \mathrm{TeV}$ [33], charm TPS takes place in about $10 \%$ of the $\mathrm{pPb}$ events at $8.8 \mathrm{TeV}$. At the FCC, the theoretical TPS charm cross section even overcomes the inclusive charm one. Such a seemingly "unphysical" result indicates that quadruple, quintuple,... parton-parton scatterings are expected to produce extra $\mathrm{c} \overline{\mathrm{c}}$ pairs with non-negligible probability in $\mathrm{pPb}$ at $\sqrt{s_{\mathrm{NN}}}=63 \mathrm{TeV}$. We recall that inclusive cross sections can be related to the factorial moments of the multiplicity distribution and, thus, SPS, DPS, TPS... cross sections are not bounded by the inelastic cross section [43]. The huge TPS c $\bar{c}$ cross sections at the FCC will make triple- $J / \psi$ production 


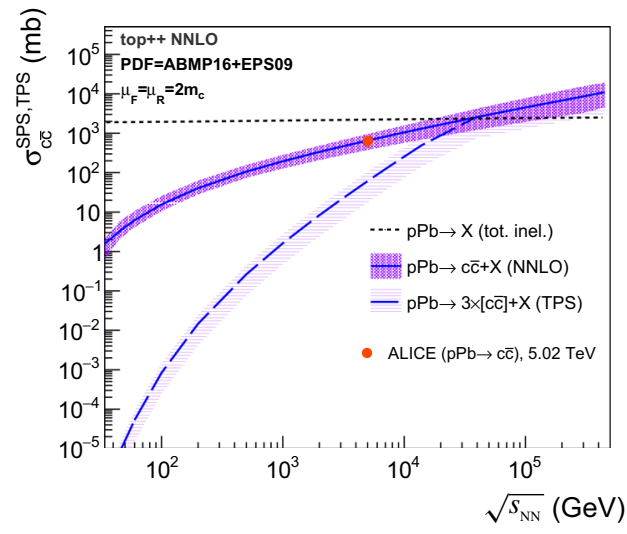

Fig. 1 Charm (left) and bottom (right) cross sections in $\mathrm{pPb}$ collisions as a function of nucleon-nucleon c.m. energy, in single-parton (solid band) and triple-parton (dashed band) scatterings, compared to the total inelastic $\mathrm{pPb}$ cross section (dotted line). Bands around curves

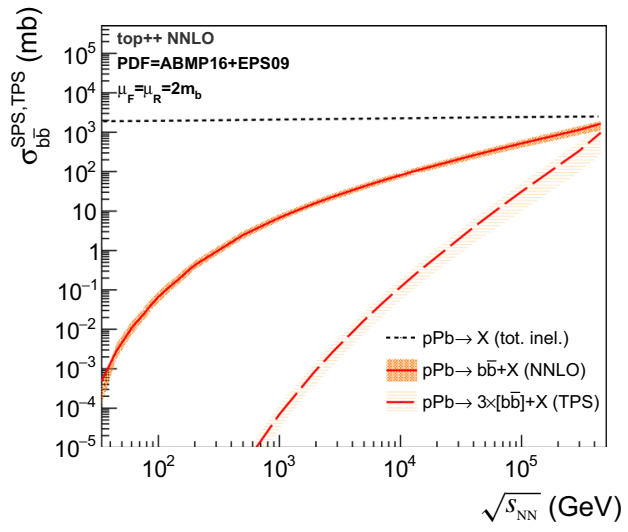

indicate scale, PDF (and $\sigma_{\text {eff,TPS }}$, in the TPS case) uncertainties added in quadrature. The $\mathrm{pPb} \rightarrow \mathrm{c} \overline{\mathrm{c}}+\mathrm{X}$ charm data point on the left plot has been derived from [42] bottom SPS (NNLO) and TPS cross sections (in $\mathrm{mb}$ ) in $\mathrm{pPb}$ at LHC and FCC energies with scales ("sc"), PDF, and total (quadratically added, including $\sigma_{\text {eff,TPS }}$ ) uncertainties
Table 1 Total charm and

\begin{tabular}{lll}
\hline Final state & $\sqrt{s_{\mathrm{NN}}}=8.8 \mathrm{TeV}$ & $\sqrt{s_{\mathrm{NN}}}=63 \mathrm{TeV}$ \\
\hline$\sigma(\mathrm{c} \overline{\mathrm{c}}+\mathrm{X})$ & $960 \pm 450_{\mathrm{sc}} \pm 100_{\mathrm{pdf}}$ & $3400 \pm 1900_{\mathrm{sc}} \pm 380_{\mathrm{pdf}}$ \\
$\sigma(\mathrm{c} \overline{\mathrm{c}} \mathrm{c} \overline{\mathrm{c}} \mathrm{c} \overline{\mathrm{c}}+\mathrm{X})$ & $200 \pm 140_{\mathrm{tot}}$ & $8700 \pm 6200_{\mathrm{tot}}$ \\
$\sigma(\mathrm{b} \overline{\mathrm{b}}+\mathrm{X})$ & $72 \pm 12_{\mathrm{sc}} \pm 5_{\mathrm{pdf}}$ & $370 \pm 75_{\mathrm{sc}} \pm 30_{\mathrm{pdf}}$ \\
$\sigma(\mathrm{b} \overline{\mathrm{b}} \mathrm{b} \overline{\mathrm{b}} \mathrm{b} \overline{\mathrm{b}}+\mathrm{X})$ & $0.084 \pm 0.045_{\mathrm{tot}}$ & $11 \pm 7_{\mathrm{tot}}$ \\
\hline
\end{tabular}

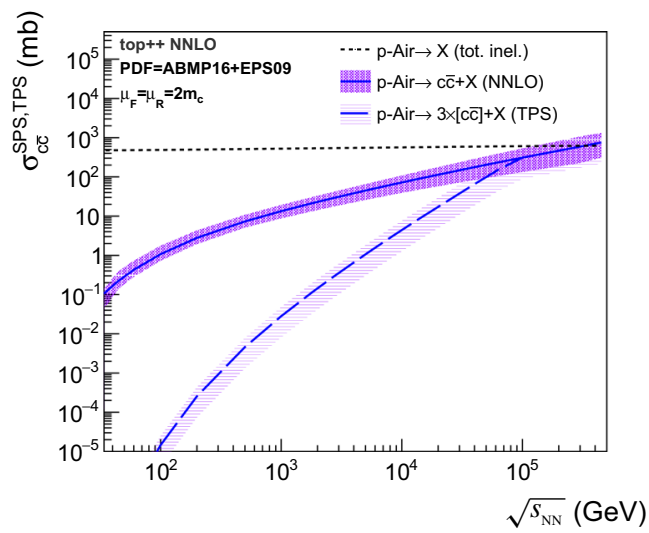

Fig. 2 Charm (left) and bottom (right) cross sections in p-Air collisions as a function of nucleon-nucleon c.m. energy, in single-parton (solid line) and triple-parton (dashed line) parton scatterings, compared

observable. Indeed, the SPS $J / \psi$ cross section corresponds to a few percent of the cc̄ one [11-14], which translates ${ }^{1}$ into $\sigma(3 \times J / \psi+\mathrm{X}) \approx 1 \mathrm{mb}$. Triple-b $\overline{\mathrm{b}}$ cross sections remain comparatively small, in the $0.1 \mathrm{mb}$ range, at the LHC but

\footnotetext{
${ }^{1}$ We note that the large production of double quarkonia seen in the data seem to indicate values of $\sigma_{\text {eff,DPs }}$ smaller than the $(15 \pm 5 \mathrm{mb})$ world-average used here [44]. Therefore, one would also expect that the corresponding TPS yields for $J / \psi$ could well be above our $1 \mathrm{mb}$ order-of-magnitude estimate.
}

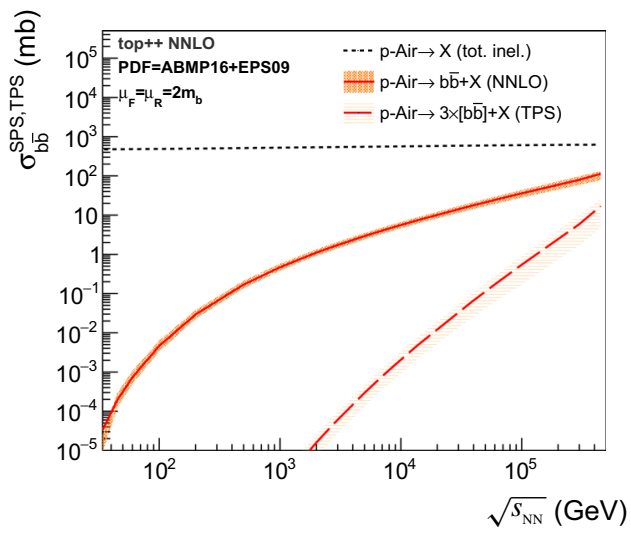

to the total inelastic p-Air cross section (dotted line). Bands around curves indicate scale, PDF (and $\sigma_{\text {eff, TPS }}$, in the TPS case) uncertainties added in quadrature

reach $\sim 10 \mathrm{mb}$ (i.e. $3 \%$ of the total inclusive bottom cross section) at the FCC.

Figure 1 shows $\mathrm{pPb}$ cross sections over $\sqrt{s_{\mathrm{NN}}} \approx 40 \mathrm{GeV}$ $100 \mathrm{TeV}$ for SPS (solid bands), TPS (dashed bands) for charm (left) and bottom (right) production, and total inelastic (dotted curve, in both plots). The TPS cross sections are small at low energies but rise fast with $\sqrt{s}$, as the cube of the SPS cross section evolution. Whenever the theoretical central value of the TPS cross section overcomes the inclusive charm cross 
section, indicative of multiple (beyond three) cc-pair production, we equalize it to the latter. Above $\sqrt{s_{\mathrm{NN}}} \approx 25 \mathrm{TeV}$, the total charm and inelastic $\mathrm{pPb}$ cross sections are equal implying that the average number of charm pairs produced in $\mathrm{pPb}$ collisions is larger than one. In the $b \bar{b}$ case, such a situation only occurs at much higher c.m. energies, above $500 \mathrm{TeV}$.

The most energetic hadronic collisions observed in nature occur in collisions of $\mathscr{O}\left(10^{20} \mathrm{eV}\right)$ cosmic rays, at the socalled "GZK cutoff" [45], with $\mathrm{N}$ and $\mathrm{O}$ nuclei at rest in the upper atmosphere. To study the amount of triple heavyquark production produced in such collisions at equivalent c.m. energies of $\sqrt{s_{\mathrm{NN}}} \approx 430 \mathrm{TeV}$, we show in Fig. 2 similar curves as those in Fig. 1 but for the p-Air case. The TPS cross sections have been obtained using Eq. (17) with the same cubic power of the SPS pN cross sections computed with the Top++ (ABMP6+EPS09) setup, but normalized now to an effective TPS p-Air cross section amounting to $\sigma_{\text {eff, } \mathrm{TPS}, \mathrm{pA}}=2.2 \pm 0.4 \mathrm{mb}$ obtained from Eq. (18) using: $\mathrm{A}=14.3$ (from a $78 \%-21 \%$ air mixture of ${ }^{14} \mathrm{~N}$ and ${ }^{16} \mathrm{O}$ ), $\mathrm{F}_{\mathrm{pA}}=0.51 \mathrm{mb}^{-1}$, and $\mathrm{C}_{\mathrm{pA}}=0.016 \mathrm{mb}^{-2}$, the latter two values being obtained via a Glauber MC [33]. Around the GZK cutoff, the cross sections for inclusive as well as TPS charm production equal the total inelastic proton-air cross section, $\sigma_{\mathrm{pAir}} \approx 0.61 \mathrm{~b}$, indicating that the average number of c $\overline{\mathrm{c}}$-pairs produced in $\mathrm{p}$-Air collisions is larger than one. In the $\mathrm{b} \overline{\mathrm{b}}$ case, about $20 \%$ of the $\mathrm{p}-\mathrm{Air}$ collisions produce bottom hadrons but only about $4 \%$ of them have TPS production. These results are clearly of relevance for the hadronic models commonly used for the simulation of the interaction of ultrarelativistic cosmic rays with the atmosphere [46] which, so far, do not include any heavy-quark production. Given that the measurements [47] observe unexplained excesses in the number of muons compared to the model predictions, and that charmed and bottom mesons feed less the non-muonic component of the air-shower, it is worth to explore the impact of properly including all such multiple heavy-quark production in the MC generators used in high-energy cosmic-ray physics.

\section{Summary}

We have derived for the first time estimates of the cross sections for triple-parton scattering (TPS) cross sections in proton-nucleus collisions as a function of the corresponding single-parton cross sections and an effective $\sigma_{\text {eff, TPS,pA }}$ parameter characterizing the transverse densities of partons in the proton and nucleus. Using NNLO predictions for single heavy-quark production, we have shown that three c $\bar{c}$-pairs are produced from separate parton interactions in $\sim 10 \%$ of the $\mathrm{pPb}$ events at the LHC. At FCC energies, more rare processes such as triple- $J / \psi$ and triple-b $\bar{b}$ production have cross sections reaching the $1-10 \mathrm{mb}$ range. At even higher energies, of a few hundred $\mathrm{TeV}$ reachable in the highest-energy collisions of cosmic rays with the nuclei in the atmosphere, the average number of c c-pairs produced in $\mathrm{p}$-Air collisions is larger than one. The quantitative results presented here are of relevance for a proper description and understanding of final states with multiple hard particles in heavy-ion collider and cosmic-ray physics at very high energies.

Acknowledgements Discussions with A.P. Kryukov and M.A. Malyshev on TPS, and with M. Cacciari, M. Czakon, A. Mitov and G. Salam on NNLO heavy-quark calculations are gratefully acknowledged.

Open Access This article is distributed under the terms of the Creative Commons Attribution 4.0 International License (http://creativecomm ons.org/licenses/by/4.0/), which permits unrestricted use, distribution, and reproduction in any medium, provided you give appropriate credit to the original author(s) and the source, provide a link to the Creative Commons license, and indicate if changes were made. Funded by SCOAP 3 .

\section{References}

1. P. Bartalini et al., arXiv: 1111.0469 [hep-ph]

2. H. Abramowicz et al., arXiv:1306.5413 [hep-ph]

3. S. Bansal et al., arXiv:1410.6664 [hep-ph]

4. R Astalos et al., arXiv:1506.05829 [hep-ph]

5. H. Jung, D. Treleani, M. Strikman, N. van Buuren, DESY-PROC2016-01

6. CDF Collaboration, Phys. Rev. D 56, 3811 (1997)

7. CMS Collaboration, Eur. Phys. J. C 76, 155 (2016)

8. ATLAS Collaboration, JHEP 11, 110 (2016)

9. LHCb Collaboration, JHEP 07, 052 (2016)

10. G. Calucci, D. Treleani, Phys. Rev. D 83, 016012 (2011)

11. D. d'Enterria, A.M. Snigirev, Phys. Lett. B 718, 1395 (2013)

12. D. d'Enterria, A.M. Snigirev, Phys. Lett. B 727, 157 (2013)

13. D. d'Enterria, A.M. Snigirev, Nucl. Phys. A 931, 296 (2014)

14. D. d'Enterria, A.M. Snigirev, Nucl. Phys. A 931, 303 (2014)

15. M. Strikman, D. Treleani, Phys. Rev. Lett. 88, 031801 (2002)

16. L. Frankfurt, M. Strikman, C. Weiss, Annalen Phys. 13, 665 (2004)

17. E. Cattaruzza, A. Del Fabbro, D. Treleani, Phys. Rev. D 70, 034022 (2004)

18. E. Cattaruzza, A. Del Fabbro, D. Treleani, Int. J. Mod. Phys. A 20, $4462(2005)$

19. A. Del Fabbro, D. Treleani, Phys. Rev. D 70, 034022 (2004)

20. A. Del Fabbro, D. Treleani, Eur. Phys. J. A 19S1, 229 (2004)

21. B. Blok, M. Strikman, U.A. Wiedemann, Eur. Phys. J. C 73, 2433 (2013)

22. G. Calucci, D. Treleani, Phys. Rev. D 79, 074013 (2009)

23. G. Calucci, D. Treleani, Phys. Rev. D 80, 054025 (2009)

24. G. Calucci, D. Treleani, Phys. Rev. D 86, 036003 (2012)

25. E. Maina, J. High Energy Phys. 09, 081 (2009)

26. A.M. Snigirev, Phys. Rev. D 94, 034026 (2016)

27. D. d'Enterria, A.M. Snigirev, Phys. Rev. Lett. 118, 122001 (2017)

28. M. Mangano, G. Zanderighi, et al., CERN-TH-2016-112, 2016; arXiv:1607.01831 [hep-ph]

29. B. Blok, Yu. Dokshitzer, L. Frankfurt, M. Strikman, Phys. Rev. D 83, 071501 (2011)

30. B. Blok, Yu. Dokshitzer, L. Frankfurt, M. Strikman, Eur. Phys. J. C 72, 1963 (2012)

31. B. Blok, Yu. Dokshitzer, L. Frankfurt, M. Strikman, Eur. Phys. J. C 74, 2926 (2014) 
32. K.J. Eskola, H. Paukkunen, C.A. Salgado, JHEP 0904, 065 (2009)

33. D. d'Enterria, nucl-ex/0302016

34. C.W. deJager, H. deVries, C. deVries, Atomic Data and Nuclear Data Tables 14 (1974) 485

35. E.R. Cazaroto, V.P. Goncalves, F.S. Navarra, arXiv:1607.04023 [hep-ph]

36. D. d'Enterria and A. Geiser, to be submitted

37. M. Czakon, P. Fiedler, A. Mitov, Phys. Rev. Lett. 110, 252004 (2013)

38. K.A. Olive et al., Particle Data Group Collaboration. Chin. Phys. C 38, 090001 (2014)

39. S. Alekhin et al., PoS DIS2016, 016 (2016), arXiv:1609.03327 [hep-ph]
40. M. Cacciari et al., JHEP 10, 137 (2012)

41. M.L. Mangano, P. Nason, G. Ridolfi, Nucl. Phys. B 373, 295 (1992)

42. ALICE Collaboration, Phys. Rev. C 94, 054908 (2016)

43. D. Treleani, G. Calucci, arXiv:1707.00271 [hep-ph]

44. J.-P. Lansberg, H.-S. Shao, Pos DIS2016, 165 (2016), arXiv:1611.02192 [hep-ph]

45. Pierre Auger Collaboration, Phys. Rev. Lett. 101, 061101 (2008)

46. D. d'Enterria, R. Engel, T. Pierog, S. Ostapchenko, K. Werner, Astropart. Phys. 35, 98 (2011)

47. Pierre Auger Collaboration Phys. Rev. D 91, 032003 (2015); Erratum: [Phys. Rev. D 91 (2015) 059901] 